\title{
Nonlocal elasticity theory for the buckling of double-layer graphene nanoribbons based on a continuum model
}

\author{
Jin-Xing Shi ${ }^{a}$, Qing-Qing Ni ${ }^{b, c^{*}}$, Xiao-Wen Lei ${ }^{a}$, Toshiaki Natsuki ${ }^{c}$ \\ a Interdisciplinary Graduate School of Science \& Technology, Shinshu University, 3-15-1 Tokida, \\ Ueda, Nagano 386-8567, Japan \\ ${ }^{\mathrm{b}}$ Key Laboratory of Advanced Textile Materials and Manufacturing Technology Ministry of \\ Education, Zhejiang Sci-Tech University, Hangzhou 310018, P. R. China \\ ${ }^{\mathrm{c}}$ Department of Functional Machinery \& Mechanics, Shinshu University, 3-15-1 Tokida, Ueda, \\ Nagano 386-8567, Japan
}

\begin{abstract}
The mechanical stability of graphene nanoribbons (GNRs) is an important mechanical property to study, when GNRs are used as components in sensors or other nanodevices. In this paper, nonlocal effects are considered in a continuum model based theoretical analysis of the critical buckling stress of cantilevered double-layer GNRs (DLGNRs) that are subjected to an axial compressive load. The results show that the nonlocal effect has an inverse relationship with the buckling stress, and the nonlocal effect decreases with increasing aspect ratio of DLGNRs. Moreover, to the best of our knowledge this is the first report that, for DLGNRs in anti-phase modes, lower buckling mode can endure higher buckling stress because of van der Waals (vdW) interaction.
\end{abstract}

Keywords: Graphene nanoribbons; Nonlocal elasticity theory; Buckling; Cantilevered beam; In-phase mode; Anti-phase mode.

* Corresponding author. Tel: 81-268-21-5438; Fax: 81-268-21-5438.

E-mail address: niqq@shinshu-u.ac.jp 


\section{Introduction}

Graphene sheet (GS), which is a single layer of carbon packed in a hexagonal (honeycomb) lattice, is the first truly two-dimensional crystalline material and was first produced in 2004 [1]. Graphene has sparked much interest among research groups recently [2-6]. Theoretically, it has a large specific surface area, high intrinsic mobility, high Young's modulus and thermal conductivity [7]. Furthermore, GS has been used as a theoretical building block of the graphite crystal and to study the formation of carbon nanotubes [8]. This two-dimensional material, which is the parent of all graphitic carbon forms, only forms single-layer atomic structures, but there is considerable interest in stacked two-layer and few-layer GSs as well [9].

Graphene nanoribbons (GNRs), are the finite-width counterparts of crystalline GSs, are interesting, because their edge shape and size control their electronic structure [5]. GNRs have been produced via chemical vapor deposition (CVD) and chemical synthesis $[8,10]$. It has also been reported that GNRs can be fabricated by unzipping carbon nanotubes (CNTs) $[11,12]$. Their outstanding mechanical, electronic transport and spin transport properties make them attractive materials for a wide range of device applications [10], such as sensors $[13,14]$. Therefore, the mechanical properties of GNRs are being actively researched.

Scholars have investigated the mechanical properties of GNRs using many methods. Frank et al. [4] showed compression behavior in monolayer GNRs through Raman spectroscopy 
using the polymer cantilever beam technique. They showed that GNRs embedded in plastic beams exhibit remarkable compression buckling strains. Xu et al. [5] presented a conformational phase diagram for rectangular GSs, defined by their geometry, boundary conditions and environmental conditions. They discovered the occurrence of three major structural arrangements and scroll phases as the aspect ratio of the GNRs increases. Lu et al. [16] showed that the excess edge energy in freestanding GNRs can be partially relaxed by both in-plane and out-of-plane deformation using a reactive empirical bond-order potential and atomistic simulations. Neek-Amal et al. [17, 18] studied the buckling behavior of monolayer GNRs subjected to axial stress both for free-boundary and supported boundary conditions using classical atomistic molecular dynamics (MD) simulations. The above mentioned references have indicated the excellent mechanical properties and pointed out the potential application of GNRs, especially in buckling behavior of GNRs, but all of them are about monolayer GNRs. However, Rao et al. [9] indicated that the properties of double-layer GSs have considerable potential for research. Thus, for layer by layer structures, such as double-layer GNRs (DLGNRs), special mechanical performance is expected because of the van der Waals (vdW) interaction between the double layers.

At a given point, the stress tensor depends linearly on the strain tensor in classical elasticity theory, which can not predict the nonlocal effects. Whereas the nonlocal elasticity theory, which assumes the stress at a reference point is considered as a function of the strain at every 
point in the body and can present the more reliable analysis. Therefore, the nonlocal effects associated nanotechnology may call the applicability of classical continuum elasticity theory into question. In especial, nonlocal effects often become prominent at nanometer scales and is deserved to study in detail. Based on the above, in the present work, we study the buckling behavior of DLGNRs that are subject to an axial compressive load by considering nonlocal effects, buckling modes and aspect ratios. Furthermore, because precisely engineered buckling geometries can be created in nanoribbons [19, 20], we intend to divide the buckling mode into in-phase and anti-phase buckling modes, which have not clearly been done and will be more important for guiding the application of DLGNRs.

\section{Theoretical approach}

GSs can be described using the discrete model [16], which is suitable for classical atomistic MD simulations. Fig. 1 (a) shows a discrete model of DLGNR with length $L$ and width $b$. Moreover, GSs can also be studied as continuum models, such as beam model [17, 18] or shell model [21-23], which are primarily used in theoretical approach. Fig. 1 (b) shows a longitudinal cross-section of the continuum DLGNR model, where $h$ is defined as the thickness of each layer of the DLGNR, which is equal to the diameter of a carbon atom, 0.34 nm. The upper and lower layers of the DLGNRs are coupled to each other via vdW interaction. 


\subsection{Governing equations}

The nonlocal elasticity theory, which could potentially play a useful role in analysis related to nanotechnology applications, has been used in the mechanical analysis of graphene sheets [22, 23], carbon nanotubes [24, 25] and other nano-sized materials [26]. Using this theory, Hooke's law for a one-dimensional stress state can be determined using the nonlinear relationship:

$$
\sigma-\left(e_{0} a\right)^{2} \frac{d^{2} \sigma}{d x^{2}}=E \varepsilon
$$

where $\sigma$ is the axial stress and $\varepsilon$ is the axial strain. $e_{0} a$ is the nonlocal parameter corresponding to each material, and $a$ is the internal characteristic length of the C-C bond which was found to be $0.142 \mathrm{~nm}$. $E$ is the elastic modulus of GNRs.

Using the definitions of the resultant bending moment and the kinematics relationship in a continuum beam model, Eq. (1) can be rewritten as:

$$
M-\left(e_{0} a\right)^{2} \frac{d^{2} M}{d x^{2}}=-E I \frac{d^{2} w}{d x^{2}}
$$

where $M$ is the resultant bending moment. $I$ is the moment of inertia of the GNR. $x$ is the longitudinal coordinate in Fig. 1 (b) and $w(x)$ is the flexural deflection of the GNR.

Based on the Euler-Bernoulli beam model, which assumes that the cross-section of a DLGNR remains planar during flexion and is perpendicular to the neutral axis, we get the following relationships: 


$$
\begin{gathered}
\frac{d V}{d x}=-p, \quad V=\frac{d M}{d x}-N \frac{d w}{d x} \\
N=2 \sigma b h
\end{gathered}
$$

where $V$ and $N$ are the resultant shear force and axial loading, respectively. $p$ is the distributed transverse pressure acting on the GNRs per unit axial length and $b$ is the width of the GNRs.

From Eqs. (2)-(4), the governing equation of motion for a continuum beam subjected to an axial load is:

$$
E I \frac{d^{4} w}{d x^{4}}+N \frac{d^{2} w}{d x^{2}}+\left(e_{0} a\right)^{2}\left(\frac{d^{2} p}{d x^{2}}-N \frac{d^{4} w}{d x^{4}}\right)-p=0
$$

For the upper and lower layers of DLGNRs, Eq. (5) can be rewritten as:

$$
\begin{aligned}
& E I \frac{d^{4} w_{1}}{d x^{4}}+\frac{N}{2} \frac{d^{2} w_{1}}{d x^{2}}+\left(e_{0} a\right)^{2}\left[b c \frac{d^{2}\left(w_{2}-w_{1}\right)}{d x^{2}}-\frac{N}{2} \frac{d^{4} w_{1}}{d x^{4}}\right]-b c\left(w_{1}-w_{2}\right)=0 \\
& E I \frac{d^{4} w_{2}}{d x^{4}}+\frac{N}{2} \frac{d^{2} w_{2}}{d x^{2}}+\left(e_{0} a\right)^{2}\left[b c \frac{d^{2}\left(w_{1}-w_{2}\right)}{d x^{2}}-\frac{N}{2} \frac{d^{4} w_{2}}{d x^{4}}\right]-b c\left(w_{2}-w_{1}\right)=0
\end{aligned}
$$

where the subscripts 1 and 2 denote the quantities associated with the upper and lower layers of the DLGNR, respectively. $c$ is the vdW interaction coefficient between the upper and lower layers, which can be obtained using the Lennard-Jones pair potential [27, 28], given by:

$$
c=-\left(\frac{4 \sqrt{3}}{9 a}\right)^{2} \frac{24 \zeta}{\sigma^{2}}\left(\frac{\delta}{a}\right)^{8}\left[\frac{3003 \pi}{256} \sum_{k=0}^{5} \frac{(-1)^{k}}{2 k+1}\left(\begin{array}{l}
5 \\
k
\end{array}\right)\left(\frac{\delta}{a}\right)^{6} \frac{1}{\left(\overline{z_{1}}-\overline{z_{2}}\right)^{12}}-\frac{35 \pi}{8} \sum_{k=0}^{2} \frac{(-1)^{k}}{2 k+1} \frac{1}{\left(\overline{z_{1}}-\overline{z_{2}}\right)^{6}}\right]
$$

where $\zeta=2.968 \mathrm{meV}$ and $\delta=3.407 \AA$ are parameters chosen to fit the physical properties of the material. $\overline{z_{l}}=z_{i} / a(i=1,2)$, where $z_{i}$ is the coordinate of the $i^{\text {th }}$ layer in the thickness direction with the origin at the mid-plane of the GNR.

To derive the in-phase and anti-phase buckling stress, we assume:

$$
\xi=w_{1}+w_{2}
$$




$$
\eta=w_{1}-w_{2}
$$

Then, from Eqs. (6) and (7), the governing equations of the in-phase and anti-phase modes are derived as:

$$
\begin{gathered}
{\left[E I-\left(e_{0} a\right)^{2} \frac{N}{2}\right] \frac{d^{4} \xi}{d x^{4}}+\frac{N}{2} \frac{d^{2} \xi}{d x^{2}}=0} \\
{\left[E I-\left(e_{0} a\right)^{2} \frac{N}{2}\right] \frac{d^{4} \eta}{d x^{4}}+\left[\frac{N}{2}+2\left(e_{0} a\right)^{2} b c\right] \frac{d^{2} \eta}{d x^{2}}-2 b c \eta=0}
\end{gathered}
$$

\subsection{Solution of governing equations}

By solving the fourth-order polynomial functional (Eqs. (11) and (12)), the solutions for the governing equations can be obtained:

$$
\begin{gathered}
\xi(x)=C_{1}+C_{2} x+C_{3} \cos \left(\lambda_{1} x\right)+C_{4} \sin \left(\lambda_{1} x\right) \\
\text { or } \quad \xi(x)=C_{1}+C_{2} x+C_{3} e^{\lambda_{2} x}+C_{4} e^{-\lambda_{2} x} \\
\text { and } \quad \eta(x)=C_{5} \cos \left(\lambda_{3} x\right)+C_{6} \sin \left(\lambda_{3} x\right)+C_{7} \cos \left(\lambda_{4} x\right)+C_{8} \sin \left(\lambda_{4} x\right) \\
\text { or } \quad \eta(x)=e^{\lambda_{5} x}\left[C_{5} \cos \left(\lambda_{6} x\right)+C_{6} \sin \left(\lambda_{6} x\right)\right]+e^{-\lambda_{5} x}\left[C_{7} \cos \left(\lambda_{6} x\right)+C_{8} \sin \left(\lambda_{6} x\right)\right] \\
\text { or } \quad \eta(x)=C_{5} e^{\lambda_{7} x}+C_{6} e^{-\lambda_{7} x}+C_{7} e^{\lambda_{8} x}+C_{8} e^{-\lambda_{8} x} \\
\text { or } \quad \eta(x)=C_{5} \cos \left(\lambda_{3} x\right)+C_{6} \sin \left(\lambda_{3} x\right)+C_{7} e^{\lambda_{8} x}+C_{8} e^{-\lambda_{8} x}
\end{gathered}
$$

where $C_{j}(j=1,2, \ldots, 8)$ are coefficients that need to be determined via boundary conditions, and

$$
\lambda_{1}=\sqrt{\frac{N}{2 E I-\left(e_{0} a\right)^{2} N}}
$$




$$
\begin{aligned}
& \lambda_{2}=\sqrt{\frac{N}{\left(e_{0} a\right)^{2} N-2 E I}} \\
& \lambda_{3}=\sqrt{\frac{\frac{N}{2}+2\left(e_{0} a\right)^{2} b c-\sqrt{\left[\frac{N}{2}-2\left(e_{0} a\right)^{2} b c\right]^{2}+8 E I b c}}{2 E I-\left(e_{0} a\right)^{2} N}} \\
& \lambda_{4}=\sqrt{\frac{\frac{N}{2}+2\left(e_{0} a\right)^{2} b c+\sqrt{\left[\frac{N}{2}-2\left(e_{0} a\right)^{2} b c\right]^{2}+8 E I b c}}{2 E I-\left(e_{0} a\right)^{2} N}} \\
& \lambda_{5}=\frac{1}{2} \\
& \lambda_{6}=\frac{1}{\frac{-N-4\left(e_{0} a\right)^{2} b c+4 \sqrt{N\left(e_{0} a\right)^{2} b c-2 E I b c}}{2 E I-\left(e_{0} a\right)^{2} N}} \\
& \lambda_{7}=\sqrt{\frac{-\frac{N}{2}-2\left(e_{0} a\right)^{2} b c-\sqrt{\left[\frac{N}{2}-2\left(e_{0} a\right)^{2} b c\right]^{2}+8 E I b c}}{2 E I-\left(e_{0} a\right)^{2} N}}
\end{aligned}
$$

\subsection{Boundary conditions}

When DLGNRs are applied in nanotechnology, two boundary conditions are in common use, which are simple supported and cantilever boundary conditions. In this study, we consider a cantilevered DLGNR subjected to an axial load $N$ with length $L$, and the corresponding boundary conditions are given as follows:

(1) for a DLGNR with fixed ends $(x=0)$ 


$$
w_{1}(0)=\frac{d w_{1}(0)}{d x}=w_{2}(0)=\frac{d w_{2}(0)}{d x}=0
$$

so we get:

$$
\xi(0)=\frac{d \xi(0)}{d x}=\eta(0)=\frac{d \eta(0)}{d x}=0
$$

(2) for a DLGNR with free ends $(x=L)$

$$
\begin{gathered}
\frac{d^{2} w_{1}(L)}{d x^{2}}=\frac{d^{2} w_{2}(L)}{d x^{2}}=0 \\
E I \frac{d^{3} w_{1}(L)}{d x^{3}}+\frac{N}{2} \frac{d w_{1}(L)}{d x}=0 \\
E I \frac{d^{3} w_{2}(L)}{d x^{3}}+\frac{N}{2} \frac{d w_{2}(L)}{d x}=0
\end{gathered}
$$

that is:

$$
\begin{gathered}
\frac{d^{2} \xi(L)}{d x^{2}}=\frac{d^{2} \eta(L)}{d x^{2}}=0 \\
E I \frac{d^{3} \xi(L)}{d x^{3}}+\frac{N}{2} \frac{d \xi(L)}{d x}=0 \\
E I \frac{d^{3} \eta(L)}{d x^{3}}+\frac{N}{2} \frac{d \eta(L)}{d x}=0
\end{gathered}
$$

Substituting the deflection functions of the DLGNR ( $\xi$ and $\eta$ ) into boundary conditions, the simultaneous equations are:

$$
\Omega[N, L]_{4 \times 4}\left[\begin{array}{l}
C_{1} \\
C_{2} \\
C_{3} \\
C_{4}
\end{array}\right]=0
$$

and

$$
\Psi[N, L]_{4 \times 4}\left[\begin{array}{l}
C_{5} \\
C_{6} \\
C_{7} \\
C_{8}
\end{array}\right]=0
$$

where $\Omega[N, L]_{4 \times 4}$ and $\Psi[N, L]_{4 \times 4}$ are $4 \times 4$ matrices for an axial compressive loading of $N$ 
and a DLGNR length of $L$. The buckling load, $N_{c}$, of the DLGNR can be obtained from the eigenvalue $|N, L|_{4 \times 4}=0$, which is the condition for a nontrivial solution of $C_{j}(j=1,2, \ldots, 8)$ in Eqs. (35) and (36).

\section{Results and Discussion}

To calculate the buckling stress of a DLGNR subjected to an axial compressive load, each layer is modeled as an individual classical thin beam of the same length, width and thickness. Assume that aspect ratio $L / b$ of each layer of a DLGNR is larger than 5 , because the Euler-Bernoulli beam theory produces a large error for short beam structures. The Young's modulus $E$ of the DLGNR, the same as that of the GSs, is $1.02 \mathrm{TPa}$ [27].

3.1 In-phase and anti-phase buckling modes

Based on this theoretical approach, buckling instability of DLGNRs with cantilevered boundary conditions can occur in both in-phase modes and anti-phase modes, which is shown in Fig. 2. This buckling instability has not been widely studied. In-phase modes 1-4 correspond to the first four buckling modes of the cantilevered DLGNRs, in which both the upper and the lower layers have the same deflection direction. Anti-phase modes 1-4 are the first four anti-phase buckling modes, in which the deflections of the upper and lower layers occur in opposite directions. 
Relationship between buckling stress and buckling modes for DLGNR of different aspect ratios with $e_{0} a=0.5 \mathrm{~nm}$ is shown in Fig. 3, in which figures (a) and (b) show buckling stress for in-phase modes 1-4 and anti-phase modes $1-4$, respectively. Fig. 3 (a) shows that the buckling stresses of the cantilevered DLGNRs with different aspect ratios all increase when the in-phase modes increase in the low buckling modes, which is the same as those in single-walled CNTs when the vdW interactions are ignored [29]. By contrast, the buckling stresses all decrease as the anti-phase buckling modes increase (see Fig. 3 (b)). Moreover, the buckling stress in anti-phase mode 1 has the highest value of the eight modes for the different lengths. We believe to this to be the first report of such behavior. This behavior arises from the vdW interaction forces between the upper and lower layers of the DLGNRs in our consideration. In addition, this behavior should be the same with simple-supported boundary condition, in which the buckling stress of DLGNRs in anti-phase mode without considering nonlocal effects can be derived as

$$
N=\frac{2 \pi^{2} E I m^{2}}{L^{2}}+\frac{-4 c L^{2}}{\pi^{2} m^{2}}
$$

where $m$ is anti-phase buckling mode.

From Eq. (37), we can clearly see that the buckling stress in anti-phase mode does not only increase with the increasing buckling mode in our common opinion, but also affected by the vdW interaction forces between the upper and lower layers of the DLGNRs. To confirm and explain this behavior in detail, we assume the vdW interaction coefficient to be $c, 10^{-1} c, 10^{-2} c$ 
and $10^{-3} \mathrm{c}$. Based on the theoretical approach in section 2, relationship between the buckling stress and anti-phase buckling modes for DLGNRs of varying vdW coefficient with $L / b=10$ and $e_{0} a=0.5 \mathrm{~nm}$ is shown in Fig. 4. We can clearly see that when vdW coefficient is assumed as $10^{-3} c$ or $10^{-2} c$, the buckling stress increases with the increasing anti-phase mode. But when the vdW coefficient is up to $10^{-1} c$ or $c$, the buckling stress decreases as the anti-phase mode increases, which means the relationship between the buckling stress and anti-phase buckling modes for DLGNRs makes a conversion when vdW coefficient is between $10^{-1} c$ and $10^{-2} c$, and can explain the larger buckling stress of the lower anti-phase modes in Fig. 3 (b) well. In reality, we need to point out that the buckling stress cannot reach such high values (see Fig. 3 (b), such as the buckling stress in anti-phase mode 1 when $L / b=20$ ), because the DLGNR subject to this axial compressive load will be damaged prior to reaching this instability. Despite this, DLGNRs can endure a much higher buckling stress in lower anti-phase modes, which is important for guiding the application of DLGNRs.

\subsection{Nonlocal effects on the buckling stress of DLGNRs}

The nonlocal effects on the buckling stress of DLGNRs in in-phase modes 1-4 and anti-phase modes 1-4 are shown in Fig. 5 (a) and (b), respectively. We choose $L / b=10$ as a representative example for the local $\left(e_{0} a=0 \mathrm{~nm}\right)$ and nonlocal $\left(e_{0} a=0.5 \mathrm{~nm}, 1.0 \mathrm{~nm}\right.$ and 2.0 $\mathrm{nm}$ ) effects on buckling stress of DLGNRs under an axial compressive load. In both in-phase 
modes and anti-phase modes, the buckling stresses decrease when $e_{0} a$ spans from 0 to $2.0 \mathrm{~nm}$. Therefore, the nonlocal effect has an inverse relationship with the buckling stress, which is in agreement with reference [24]. This effect is most prominent in higher in-phase modes and lower anti-phase modes. In particular, for in-phase mode 1, the buckling stresses of the DLGNRs under local and nonlocal effects are nearly the same. Furthermore, when $e_{0} a=2.0$ nm, only in-phase modes 1 and 2 exist with $L / b=10$, because the aspect ratio is not large enough to produce the higher-order modes. This phenomena also occurs when $e_{0} a=2.0 \mathrm{~nm}$ (see Fig. 5 (a)). In this case, the DLGNRs do not have an in-phase mode for $L / b=5$.

The nonlocal effects on the buckling stress versus aspect ratio are also discussed in this study. The relationship between buckling stress and the aspect ratio of the DLGNR for in-phase mode 2 is shown in Fig. 6. Similar to the case in Fig. 5, the nonlocal effect has an inverse relationship with the buckling stress. With increasing DLGNR aspect ratio, the nonlocal effect decreases, which is in agreement with reference [30]. For example, when the aspect ratio of a DLGNR is 20, for $e_{0} a$ values from $0-2 \mathrm{~nm}$, the critical buckling stresses change within $0.13 \mathrm{GPa}$, which illustrate that the four different buckling stresses are similar to each other in high aspect ratio.

\section{Conclusions}

An analytical procedure based on the Euler-Bernoulli beam model was used to investigate 
the buckling instability due to nonlocal effects on DLGNRs subjected to an axial compressive load. The buckling modes of DLGNRs were divided into in-phase and anti-phase modes, in which the deflections of the upper and lower layers occur in the same direction and the opposite directions, respectively. Moreover, to the best of our knowledge, this is the first report that the buckling stresses decrease as the anti-phase buckling modes increase, which means buckling stress for DLGNRs in lower anti-phase mode owns higher buckling stress. The influence of the nonlocal effects on the buckling stress were also discussed in detail. The results show that the nonlocal effect has an inverse relationship with the buckling stress, and the nonlocal effect decreases with increasing DLGNR aspect ratio. This investigation will be helpful for DLGNR applications such as buckling mechanical analysis, mechanical sensors and graphene-based electrochemical sensors.

\section{Acknowledgments}

This work was supported by a Grant-in-Aid from the Global COE Program from the Ministry of Education, Culture, Sports, Science and Technology and by CLUSTER (second stage) from the Ministry of Education, Culture, Sports, Science and Technology (Japan).

\section{References}

[1] K.S. Novoselov, A.K. Geim, S.V. Morozov, D. Jiang, Y. Zhang, S.V. Dubonos, I.V. 
Grigorieva, A.A. Firsov, Electric field effect in atomically thin carbon films, Science 306 (2004) 666-669.

[2] J.C. Meyer, A.K. Geim, M.I. Katsnelson, K.S. Novoselov, T.J. Booth, S. Roth, The structure of suspended graphene sheets, Nature 446 (2007) 60-63.

[3] M.J. McAllister, J.L. Li, D.H. Adamson, H.C. Schniepp, A.A. Abdala, J. Liu, M. Herrera-Alonso, D.L. Milius, R. Car, R.K. Prud'homme, I.A. Aksay, Single sheet functionalized graphene by oxidation and thermal expansion of graphite, Chem. Mater. 19 (2007) 4396-4404.

[4] H.C. Schniepp, K.N. Kudin, J.L. Li, R.K. Prud'homme, R. Car, D.A. Saville, I.A. Aksay, Bending properties of single functionalized graphene sheets probed by atomic force microscopy, ACS-Nano. 2 (2008) 2577-2584.

[5] Z.P. Xu, M.J. Buehler, Geometry controls conformation of graphene sheets: membranes, ribbons, and scrolls, ACS-Nano. 4 (2010) 3869-3876.

[6] R. Grantab, V.B. Shenoy, R.S. Ruoff, Anomalous strength characteristics of tilt grain boundaries in grapheme, Science 330 (2010) 946-948.

[7] Y.W. Zhu, S. Murali, W.W. Cai, X.S. Li, J.W. Suk, J.R. Potts, R.S. Ruoff, Graphene and graphene oxide: synthesis, properties, and applications, Adv. Mater. 22 (2010) 3906-3924.

[8] M. Terrones, A.R. Botello-Méndez, J. Campos-Delgado, F. López-Urías, Y.I. Vega-Cantú, F.J. Rodríguez-Macías, A.L. Elías, E. Muñoz-Sandoval, A.G. Cano-Márquez, J.C. Charlier, H. 
Terrones, Graphene and graphite nanoribbons: morphology, properties, synthesis, defects and applications, Nano Today 5 (2010) 351-372.

[9] C.N.R. Rao, A.K. Sood, K.S. Subrahmanyam, A. Govindaraj, Graphene: The new two-dimensional nanomaterial, Angew. Chem. Int. Ed. 48 (2009) 7752-7777.

[10] M.A. Rafiee, W. Lu, A.V. Thomas, A. Zandiatashbar, J. Rafiee, J.M. Tour, N.A. Koratkar, Graphene nanoribbon composites, ACS-Nano 4 (2010) 7415-7420.

[11] L.Y. Jiao, L. Zhang, X.R. Wang, G. Diankov, H.J. Dai, Narrow graphene nanoribbons from carbon nanotubes, Nature 458 (2009) 877-880.

[12] D.V. Kosynkin, A.L. Higginbotham, A. Sinitskii, J.R. Lomeda, A. Dimiev, B.K. Price,. J.M. Tour, Longitudinal unzipping of carbon nanotubes to form graphene nanoribbons, Nature 458 (2009) 872-876.

[13] B. Huang, Z.Y. Li, Z.R. Liu, G. Zhou, S.G. Hao, J. Wu, B.L. Gu, W.H. Duan, Adsorption of gas molecules on graphene nanoribbons and its implication for nanoscale molecule sensor, J. Phys. Chem. C 112 (2008) 13442-13446.

[14] M.S.G.M. Pumera, Graphene-based electrochemical sensor for detection of 2,4,6-trinitrotoluene (TNT) in seawater: the comparison of single-, few-, and multilayer graphene nanoribbons and graphite microparticles, Anal. Bioanal. Chem. 399 (2011) 127-131.

[15] J.L. Johnson, A. Behnam, S.J. Pearton, A. Ural, Hydrogen sensing using 
pd-functionalized multi-layer graphene nanoribbon networks, Adv. Mater. 22 (2010) 4877-4880.

[16] Q. Lu, R. Huang, Excess energy and deformation along free edges of graphene nanoribbons, Phys. Rev. B 81 (2010) 155410-1-7.

[17] M. Neek-Amal, F.M. Peeters, Graphene nanoribbons subjected to axial stress, Phys. Rev. B 82 (2010) 085432-1-6.

[18] M. Neek-Amal, F.M. Peeters, Defected graphene nanoribbons under axial compression, Appl. Phys. Lett. 97 (2010) 153118-1-3.

[19] Y.G. Sun, W.M. Choi, H.Q. Jiang, Y.G. Huang, J.A. Rogers, Controlled buckling of semiconductor nanoribbons for stretchable electronics, Nat. Nanotechnol 1 (2006) 201-207.

[20] H.Q. Jiang, J.A. Rogers, Y.G. Huang, Mechanics of precisely controlled thin film buckling on elastomeric substrate, Appl. Phys. Lett. 90 (2007) 133119-1-3.

[21] D.D. Quinna, J.P. Wilber, C.B. Clemons, G.W. Young, A. Buldum, Buckling instabilities in coupled nano-layers, Int. J. Non-Linear Mech. 42 (2007) 681-689.

[22] S.C. Pradhan, Buckling of single layer graphene sheet based on nonlocal elasticity and higher order shear deformation theory, Phys. Lett. A 373 (2009) 4182-4188.

[23] S.C. Pradhan, T. Murmu, Small scale effect on the buckling of single-layered graphene sheets under biaxial compression via nonlocal continuum mechanics, Comp. Mater. Sci. 47 (2009) 268-274. 
[24] Q. Wang, V.K. Varadan, S.T. Quek, Small scale effect on elastic buckling of carbon nanotubes with nonlocal continuum models, Phys. Lett. A 357 (2006) 130-135.

[25] M.J. Hao, X.M. Guo, Q. Wang, Small-scale effect on torsional buckling of multi-walled carbon nanotubes, Eur. J. Mech. A/Solids 29 (2010) 49-55.

[26] J.K. Phadikar, S.C. Pradhan, Vibrational formulation and finite element analysis for nanobeams and nanoplates, Comp. Mater. Sci. 49 (2010) 492-499.

[27] S. Kitipornchai, X.Q. He, K.M. Liew, Continuum model for the vibration of multilayered graphene sheets, Phys. Rev. B 72 (2005) 075443-1-6.

[28] K.M. Liew, X.Q. He, S. Kitipornchai, Predicting nanovibration of multi-layered graphene sheets embedded in an elastic matrix, Acta Mater. 54 (2006) 4229-4236.

[29] B.I. Yakobson, C.J. Brabec, J. Bernholc, Nanomechanics of carbon tubes: instabilities beyond linear response, Phys. Rev. Lett. 76 (1996) 2511-2514.

[30] J.N. Reddy, Nonlocal theories for bending, buckling and vibration of beams, Int. J. Eng. Sci. 45 (2007) 288-307. 


\section{Figure captions}

Fig. 1 Analytical model for cantilevered DLGNRs, (a) Discrete model and (b) Longitudinal cross-section of the continuum model.

Fig. 2 Buckling modes of cantilevered DLGNRs.

Fig. 3 Relationship between the buckling stress and buckling modes for DLGNRs of varying aspect ratio with $e_{0} a=0.5 \mathrm{~nm}$, (a) In-phase modes and (b) Anti-phase modes.

Fig. 4 Relationship between the buckling stress and anti-phase buckling modes for DLGNRs of varying vdW coefficient with $L / b=10$ and $e_{0} a=0.5 \mathrm{~nm}$.

Fig. 5 Relationship between the nonlocal effect and buckling stress of DLGNRs for $L / b=10$ in different buckling modes. (a) In-phase modes and (b) Anti-phase modes.

Fig. 6 Relationship between the nonlocal effect and buckling stress for DLGNRs of varying aspect ratio for in-phase mode 2. 